\title{
Author Correction: Dopamine transients are sufficient and necessary for acquisition of model-based associations
}

Melissa J Sharpe, Chun Yun Chang, Melissa A Liu, Hannah M Batchelor, Lauren E Mueller, Joshua L Jones, Yael Niv D and Geoffrey Schoenbaum (D)

Correction to: Nature Neuroscience https://doi.org/10.1038/nn.4538, published online 3 April 2017.

In the version of this article initially published, the laser activation at the start of cue $\mathrm{X}$ in experiment 1 was described in the first paragraph of the Results and in the third paragraph of the Experiment 1 section of the Methods as lasting $2 \mathrm{~s}$; in fact, it lasted only $1 \mathrm{~s}$. The error has been corrected in the HTML and PDF versions of the article.

\section{Author Correction: Synaptic $N^{6}$-methyladenosine $\left(\mathrm{m}^{6} \mathrm{~A}\right)$ epitranscriptome reveals functional partitioning of localized transcripts \\ Daria Merkurjev, Wan-Ting Hong, Kei lida, Ikumi Oomoto, Belinda J. Goldie (D), Hitoshi Yamaguti, Takayuki Ohara, Shin-ya Kawaguchi (D, Tomoo Hirano, Kelsey C. Martin, Matteo Pellegrini and Dan Ohtan Wang (D)}

Correction to: Nature Neuroscience https://doi.org/10.1038/s41593-018-0173-6, published online 27 June 2018.

In the version of this article initially published, a Supplementary Fig. $6 \mathrm{f}$ was cited in the last paragraph of the Results. No such panel exists; the citation has been deleted. The error has been corrected in the HTML and PDF versions of the article.

\section{Author Correction: Retrieval induces adaptive forgetting of competing memories via cortical pattern suppression}

Maria Wimber, Arjen Alink, lan Charest, Nikolaus Kriegeskorte and Michael C Anderson

Correction to: Nature Neuroscience https://doi.org/10.1038/nn.3973, published online 16 March 2015.

In the published version of this article, a detail is missing from the Methods section "Experimental procedure." The following sentence is to be inserted at the end of its fourth paragraph: "If participants failed to respond within $3.5 \mathrm{~s}$, we assumed that they were unable to successfully recognize the item and coded the corresponding trial as an error." The critical behavioral forgetting effect is significant irrespective of whether these timeouts are coded as errors $\left(t_{23}=4.91, P<0.001\right)$ or as missing data $\left(t_{23}=3.31, P<0.01\right)$. The original article has not been corrected. 